\title{
An update on the treatment of canine atopic dermatitis
}

This article was published in the following Dove Press journal:

Veterinary Medicine: Research and Reports

29 August 2012

Number of times this article has been viewed

\section{Rosanna Marsella \\ Department of Small Animal Clinical Sciences, College of Veterinary Medicine, University of Florida Gainesville, FL, USA}

\begin{abstract}
Remarkable progress has been made in recent years concerning our understanding of the pathogenesis of canine atopic dermatitis (AD). As our understanding improves, the therapeutic approach evolves. Of utmost importance is the documentation of skin barrier impairment in canine AD: ceramides deficiency leads to increased permeability and increased allergen penetration and sensitization. It is currently unknown whether this dysfunction is primary and genetically inherited or secondary to inflammation but it is accepted that skin barrier deficiency plays an important role in either starting or minimally exacerbating canine AD. Thus, the therapeutic approach has changed from focusing on the control of the inflammation to a combined approach that includes therapies aimed at skin barrier repair. The issue of skin barrier repair has been addressed both with oral administration of essential fatty acids and the topical application of products containing a combination of ceramides and fatty acids. These strategies are most helpful as adjunctive treatments and would be best used in young patients that have not developed chronic skin changes. Importantly, treatment for canine AD is multimodal and tailored to the individual patient, the age, and the duration of the disease. Client education plays an important role in explaining the importance of a long-term approach to minimize flare-ups and, in this context, topical therapy to correct skin barrier can be of great benefit. This is an area still in infancy and much work is needed to identify the best formulation. In human medicine, long-term use of moisturizers can have a profound effect on skin barrier and gene expression of proteins involved in skin barrier. This effect is variable depending on the formulation used. It is reasonable to speculate that the same may be true in dogs; thus, it is very important to identify the correct ingredients and formulation to use.
\end{abstract}

Keywords: dogs, atopic dermatitis, treatment, skin barrier repair

\section{Introduction}

Canine atopic dermatitis (AD) is a chronic relapsing pruritic skin disease that results from the complex interaction between genetics and environmental factors. The challenge in completely understanding and successfully managing this condition comes from the fact that canine AD is most likely a clinical syndrome rather than a single disease. Many patients that present with clinical signs compatible with AD may have different mechanisms of disease and may respond differently to treatments. At this point, we do not have tests to identify which specific mutation/mechanism is most relevant in each individual patient and prescribe treatments accordingly. Thus, historically, the most commonly used and effective treatments have been broad-spectrum therapies such as glucocorticoids. These agents are able to suppress a multitude of inflammatory mediators, effectively providing relief from the
Correspondence: Rosanna Marsella Department of Small Animal Clinical Sciences, University of Florida, 2015 SW 16th Avenue, Gainesville, FL 32610, USA

Tel +l 3522780742

Fax +l 3523926125

Email marsella@ufl.edu 
allergic response in most patients, at least in the short term. Traditionally, this has been the main approach to AD, but, since this disease is chronic in nature, it is very important to provide alternative options as the efficacy of glucocorticoids tends to wane over time and adverse effects may become unacceptable. For these reasons, it is important to have a long-term approach as well as strategies to cope with the acute flare-ups and with the secondary infections, which constitute a significant aggravating factor. With the increased frequency of antibiotic resistance in veterinary medicine, ${ }^{1}$ the chronic use of antibiotics should be minimized and alternative approaches to deal with the increased staphylococcal colonization $^{2}$ of these patients becomes crucial. In light of this consideration, topical therapy is extremely important and should be considered as an integral part of the longterm management. Recent studies have also highlighted the importance of skin barrier impairment in canine AD. ${ }^{3}$ Skin barrier dysfunction can lead to increased allergen penetration and increased risk for allergic sensitization. For this reason, any long-term plan should also consider strategies aimed at either improving or at least minimizing any damage to the skin. Although this area has not been fully elucidated, and evidence of efficacy is still preliminary, approaches to restore and nurture the skin should be considered in the approach to any case of canine AD.

\section{Treatment options and evidence of efficacy}

A systematic review of treatments used for canine AD analyzed the results of randomized controlled studies published from January 1980 to December 2007 and found evidence of efficacy for topical tacrolimus, topical triamcinolone, oral glucocorticoids, oral cyclosporine, subcutaneous recombinant gamma-interferon, and subcutaneous allergen-specific immunotherapy to decrease pruritus and/or skin lesions of $\mathrm{AD}$ in dogs. ${ }^{4}$ Of these options, some are used for the management of the acute flare-up and others are best suited for the long-term approach.

\section{Approach to the acute flare-up}

Many dogs with AD have an allergic component and flareups are common when allergens to which they are sensitized are most prevalent. These flare-ups may just be allergic in nature or complicated by secondary infections. According to the practice guidelines from the International Task Force for Canine $\mathrm{AD},{ }^{5}$ acute flares should be treated with a combination of nonirritating baths and topical glucocorticoids, and effort should be made to identify and correct the triggering factors.
Many atopic patients have multiple sensitizations and flare factors range from foods to environmental allergens and insects. Thus, the approach to these cases has to be multimodal, correcting, and minimizing any factors that can push the patient over the threshold of clinical disease. Indeed, according to the threshold of pruritus, individuals are able to tolerate a certain amount of pruritic stimuli without showing clinical signs. Once that threshold is exceeded, clinical signs develop. This concept is of critical importance in the clinical management of dogs with AD. Thus, when an acute flare-up occurs, it is important to understand the reasons and correct them as much as possible.

It is important to realize that the traditional and somewhat artificial separation between "food allergy" or a food-induced dermatitis and AD is no longer convincing. Food-induced dermatitis can manifest in many ways and, in some patients, it can manifest as the clinical syndrome of AD. ${ }^{6}$ These patients look clinically indistinguishable from other patients with AD in which the disease is triggered by environmental allergens or from patients that have no detectable allergen-specific immunoglobulin E (IgE; " atopic-like $\mathrm{AD}$ or intrinsic $\left.\mathrm{AD}^{\prime \prime}\right)^{7,8}$. For these reasons, it is important to investigate the role played by foods in all cases of AD, particularly the ones that are nonseasonal. The concept of patients with $\mathrm{AD}$ in the absence of allergic sensitization will be addressed later under the section on intrinsic AD.

Acute inflammation can be addressed with glucocorticoids either topically or orally as a short course. Topical treatment is safer than systemic due to the minimal absorption and the oral route is preferred over the injectable as it allows tapering schedules with alternate day administration, which helps to minimize adrenal suppression. Injectable formulations should therefore be avoided when managing patients with $\mathrm{AD}$. In a placebo-controlled clinical trial, topical $0.0584 \%$ hydrocortisone aceponate spray was shown to be rapidly effective in controlling pruritus in dogs with AD. ${ }^{9}$ In this study, clinical scores and pruritus were significantly decreased after 2 weeks of therapy and no adverse effects were reported. In another study comparing topical hydrocortisone aceponate spray with oral cyclosporine, no significant difference was found between treatments in terms of clinical efficacy. ${ }^{10} \mathrm{~A}$ low percentage of triamcinolone applied topically is another option to effectively and rapidly control pruritus in atopic dogs. ${ }^{11}$ This treatment modality is safe and well tolerated in most cases.

Bathing with nonirritating shampoos also provides temporary relief to pruritic dogs and is an important component of therapy in both acute and chronic management of dogs with 
AD. Interestingly, in a recently published study, a medicated shampoo containing essential fatty acids was as effective as its vehicle. ${ }^{12}$ This outcome may be explained by the fact that washing in itself helps to removes the allergen from the coat. Since the epicutaneous route of allergen exposure is one of the most important ways by which dogs are exposed to the allergen, ${ }^{13}$ bathing with nonirritating products is of great benefit both for the acute flare-ups and in the long-term management. Another study comparing conventional shampoo therapy of pruritic dogs with shampoo therapy applied in a whirlpool and water therapy in a whirlpool reported significant improvement of clinical scores in the group allocated to shampoo therapy in the whirlpool compared to conventional shampooing. The authors concluded that conventional shampoo therapy is more effective as a treatment for pruritus than water alone and that shampoo therapy in the whirlpool is more effective as an antipruritic therapy than water alone, proposing that whirlpooling may be a useful treatment option to help pruritic dogs. ${ }^{14}$ If the acute flare-up is associated with a secondary infection, this needs to be properly diagnosed and treated, as infections significantly contribute to the level of discomfort. Milder cases may be treated with topical antimicrobials while more severe cases require systemic courses of antibiotics. To minimize antibiotic resistance, the choice of the antibiotic and the length of therapy are of critical importance.

\section{Long-term approach to chronic cases}

In dogs with chronic $\mathrm{AD}$, a combination of interventions is necessary and needs to take into account both the control of the secondary infections and the underlying AD. As in the acute cases, all flare factors need to be identified and corrected. Chronic recurrent bacteria and yeast infections are very common in these patients. Regular topical antimicrobial therapy is essential and may be alternated with topical therapy to alleviate pruritus. Besides the traditional ingredients used (eg, benzoyl peroxide and chlorhexidine), new approaches are considered in patients with staphylococcal infections. In humans, diluted bleach baths are used to decrease the carriage of Staphylococcus ${ }^{15}$ in patients with AD. ${ }^{16,17}$ In veterinary medicine, the use of products containing oxychlorine (Vetericyn; Innovacyn, Rialto, CA) is becoming popular. Although randomized controlled clinical trials are lacking in veterinary medicine, anecdotal reports suggest that topical oxychlorine is quite effective as an adjunctive treatment for bacterial infections. The management of the recurrent bacterial infections in dogs with $\mathrm{AD}$ is becoming a major challenge due to multidrug resistance; thus, it is important for clinicians to minimize antibiotic use and to favor narrow spectrum antibiotics over broad spectrum. Proper duration and appropriate doses are crucial for success to minimize treatment failures or relapses.

Due to the chronicity of $A D$, every effort should be made to limit the use of treatment options for $A D$ that may predispose to infections (eg, minimizing the use of glucocorticoids) and favor approaches that are sustainable in the long term (eg, allergen-specific immunotherapy). Allergen-specific immunotherapy (allergy vaccine) should be attempted whenever possible, particularly if symptoms are present for more than a few months/year. This form of therapy should not be considered only after all treatments have failed and after years of chronic disease. On the contrary, it should be considered in young patients, particularly if living in areas of high pollen load. Success with this approach is better in younger individuals and depends on the skills of the clinician in selecting allergens that are clinically relevant. For this reason, it is important to correlate the results of the allergy testing with the seasonality of the patient and presence of those allergens in the environment. Careful selection of allergens seems to be more important than the specific allergy test used (eg, serology testing vs intradermal skin testing).

Allergen-specific immunotherapy has on average a success rate ranging from $60 \%-80 \%$, where success typically means fewer relapses and less need for rescue medications but does not imply a cure. ${ }^{18,19}$ Most cases require long-term allergy vaccines and it is not uncommon to change the composition of the vaccine every couple of years to reflect changes in the sensitization pattern of the patient. While historically the allergy vaccine in veterinary medicine has been administered by subcutaneous injections, there is preliminary evidence that sublingual administration of allergen ${ }^{20}$ is well tolerated and similarly effective as injectable immunotherapy in dogs with AD. This area of research is new in veterinary medicine and will benefit from larger controlled clinical trials to identify the best treatment schedules.

Various schedules have been used by clinicians to build up the amount of allergen given in the injectable vaccines. Controlled studies to objectively compare different schedules are scarce; ${ }^{21}$ thus, allergen-specific immunotherapy is still considered more an art than a science. Adjusting the schedule to suit the needs of the patient is crucial to maximize success and minimize adverse effects and requires education and consistent communication with the owners.

Many clinicians incorporate the use of antihistamines in the long-term regimens of their atopic patients. 
Interestingly, insufficient evidence was found to support the use of antihistamines for canine AD in a systematic review. ${ }^{22}$ This result may derive from the stringent criteria used in the review. As antihistamines are best used as adjunctive therapy, it is understandable why they would not reach sufficient efficacy to be recommended when they are tested as monotherapy. As a matter of fact, a recent publication reported that owners perceived clinical benefit from the use of antihistamines in their dogs. ${ }^{23}$ The discrepancy in findings may stem from the criteria used in the two studies and the difference in assessment between investigators and owners. It is important to emphasize the concept that although antihistamines may not be a sufficiently strong treatment to help dogs with AD as monotherapy, many practitioners feel that they have a place to help mild-to-moderate cases, particularly as adjunctive treatment to minimize the need for rescue medications such as glucocorticoids. The same concept applies to essential fatty acids, which also were not found to be effective as monotherapy in the systematic review of treatments for canine $\mathrm{AD} .{ }^{4}$ Yet, in clinical practice, they are frequently used as adjunctive therapy. The benefit of essential fatty acids in canine AD may be due to a multitude of properties ranging from modulation of the inflammation to the repair of skin barrier. In a recently published study, ${ }^{24}$ 3 weeks of oral administration of essential fatty acids led to improvement of the ultrastructure of the skin and normalization of some of the chemical aberrations of the upper layers of the epidermis, suggesting a beneficial effect of this form of therapy. Additional investigation is needed to assess the correlation between improvement of the skin barrier alterations and amelioration of clinical signs in order to identify the most beneficial way to use essential fatty acids as part of the management of dogs with AD.

Pentoxifylline was found to have fair evidence of efficacy in the systematic review of treatments for AD. ${ }^{4}$ Yet, a more recent study ${ }^{25}$ using this medication at higher doses $(20 \mathrm{mg} / \mathrm{kg}$ three times daily versus $10 \mathrm{mg} / \mathrm{kg}$ twice daily of the original studies ${ }^{26}$ ) and in combination with essential fatty acids showed a marked beneficial effect. In this study, dogs receiving the combination of pentoxifylline and essential fatty acids revealed $56.75 \%$ and $47.76 \%$ reduction in clinical scores and pruritus scores on day 30 , while the corresponding reduction on day 60 was $90.92 \%$ and $92.59 \%$, respectively. It is therefore reasonable to conclude that pentoxifylline is a beneficial adjunctive treatment (eg, in combination with essential fatty acids) for canine $\mathrm{AD}$, especially to reduce the frequency of administration of glucocorticoids.

\section{Skin barrier repair treatments}

The investigation of the role of skin barrier dysfunction in canine $\mathrm{AD}$ is relatively new. While in human medicine, this aspect of the disease has been known for a long time and topical use of emollients and moisturizers is the mainstay of therapy, ${ }^{27}$ in dogs, skin barrier repair is a novel area of research. Skin barrier impairment in dogs with AD has been described ultrastructurally, ${ }^{28}$ chemically, ${ }^{29,30}$ and functionally ${ }^{31}$ through the measurement of transepidermal water loss (TEWL), yet the exact significance of it is still an object of controversy. A recently published study failed to find a correlation between TEWL and severity of clinical signs as measured by the validated Canine Atopic Dermatitis Severity Index (CADESI) score. ${ }^{32}$ This finding in itself does not minimize the clinical relevance of the skin barrier dysfunction in dogs with AD: the lack of correlation may be due to either the methodology used for the measurement of TEWL or the validity of CADESI. Regardless of the reason, this lack of obvious correlation makes the issue of the assessment of clinical relevance of skin barrier impairment more complex, particularly when this is a methodology used to evaluate the improvement of various treatment options in clinical trials. TEWL measurements have been shown to vary from site to site and from day to day, ${ }^{33}$ thus it is difficult to assess the beneficial effect of skin repair treatments relying only on measurements of TEWL. Measurement of TEWL as an indicator of skin barrier function is appealing as it is noninvasive but ultimately it may be better to assess repair of skin barrier in a variety of ways, ranging from biopsy for electron microscopy studies to chemical analysis of lipid composition of the stratum corneum. Indeed, in a recent study, 3 weeks of topical administration of an emulsion containing ceramides, free fatty acids, and cholesterol (skin lipid complex) led to significantly increased values for ceramides. ${ }^{34}$ Although in this study no assessment of clinical improvement was done, in a subsequent open clinical trial using the same preparation twice weekly for 12 weeks, improvement of clinical signs was reported. ${ }^{35}$ In this study, topical application of the ceramide-based "spot-on" (Allerderm Spot On ${ }^{\circledR}$; Virbac, Fort Worth, TX) helped to decrease clinical signs of AD in dogs that had failed to respond to other therapies. The beneficial effect was most evident after several weeks of therapy with a statistically significant reduction of CADESI and specific reduction of erythema from the baseline after 6 weeks. The authors concluded that this treatment modality is best used as adjunctive therapy. Another yet unpublished study evaluating the same formulation in a blinded and controlled fashion confirmed the beneficial effect in canine $\mathrm{AD}^{36}$ after 
4 weeks of application. Thus, it is reasonable to believe that topical application of ceramides may have a place in the management of canine patients with $\mathrm{AD}$, as has been the case in humans with AD. ${ }^{37,38}$ Indeed, lipid-rich topical preparations in humans with $\mathrm{AD}$ have been found to be as effective as topical glucocorticoids, ${ }^{39}$ but without the unwanted adverse effect of cutaneous atrophy that long-term application of glucocorticoids can cause.

Limited studies have also been done to investigate the efficacy of a topical preparation containing phytosphingosine, a ceramides precursor. A blinded randomized controlled trial in privately owned dogs with AD using either a phytosphingosine-containing shampoo (Douxocalm ${ }^{\circledR}$; Sogeval, Irving, $\mathrm{TX}$ ), a phytosphingosine-containing shampoo plus spray with similar ingredients, or a control shampoo containing antiseptics, fatty acids, and complex sugars (Allermyl ${ }^{\circledR}$; Virbac $)^{40}$ showed clinical improvement in both groups. Since all interventions were theoretically able to affect skin barrier, it is unknown whether using phytosphingosine provides extra benefit compared to any emollient or simple bathing.

\section{Intrinsic AD or atopic-like dermatitis}

The concept of dogs that may have clinical signs of AD in the absence of an allergic sensitization (so-called intrinsic form) is relatively recent. Intrinsic disease has been known in human medicine for decades and it seems to represent a separate subset of disease in which skin barrier is not impaired and clinical signs are milder and delayed in onset. ${ }^{41}$ In veterinary medicine, cases clinically diagnosed with $\mathrm{AD}$ in which allergen-specific IgE cannot be demonstrated are occasionally described. An interesting study evaluating the effect of 2-week isolation in 27 dogs with clinical diagnosis of AD showed that $63 \%$ of them improved while the rest did not. ${ }^{42}$ The improvement was considered most likely due to the allergen avoidance in the hospital environment without furniture and carpets. Among the remaining 37\%, 14\% (4/27) of these dogs had a negative intradermal allergy testing for all environmental antigens. Dogs for which sensitivity to an environmental antigen was not identified were identified to have atopic-like dermatitis. These dogs represent a therapeutic challenge as triggering factors cannot be identified.

\section{Can AD be prevented?}

Although AD has a strong genetic basis, the interaction with the environment determines the final outcome. It has been observed that $\mathrm{AD}$ has increased dramatically in the last few decades in industrialized countries and, according to the hygiene theory, ${ }^{43,44}$ this increase is, at least in part, determined by changes in lifestyle conditions. These changes include a decreased exposure to beneficial bacteria that have protective effects in the modulation of the immune system. For this reason, great interest has been placed in the prophylactic use of probiotics in individuals at high risk for AD ${ }^{45,46}$ Although the results of the effects of probiotic exposure in human medicine have been varied, it is accepted that the best use of probiotics is indeed preventative rather than treatment of an already-established disease. The same may be true in veterinary medicine. A pilot study using an experimental model showed that early exposure to Lactobacillus rhamnosus GG could decrease allergen-specific IgE and severity of clinical signs. ${ }^{47}$ The study was done using an experimental model of canine $\mathrm{AD}$ and the exposure to probiotics was done prenatally by administration to the dam during gestation and postnatally for the first 6 months of life. A follow-up study 3 years after discontinuation of probiotics showed that the protective effect in those dogs was still present and was associated with a modulation of $\mathrm{T}$ regulatory lymphocytes. ${ }^{48}$ Thus, although these are pilot studies, it is encouraging that modulation of the immunologic response using probiotics in individuals at high risk is possible and may have long-lasting protective effects.

\section{Conclusion}

Canine AD is a prevalent and chronic clinical syndrome with a complex pathogenesis, which may be different from patient to patient. The management of AD should be multimodal to control both the underlying inflammation and the secondary complicating infections. Treatment should include long-term strategies as well as a plan to address the acute flare-ups. Care should be used in the selection of topical therapy since preliminary evidence indicates that skin barrier dysfunction plays an important role in canine AD. Additional studies are necessary to identify effective treatments to be used for skin barrier repair.

\section{Disclosure}

The author has received funding for studies from Novartis, Pfizer, Sogeval, Virbac, Boehringer, and Merial. The author does not have any stock in any pharmaceutical company.

\section{References}

1. Chrobak D, Kizerwetter-Swida M, Rzewuska M, Binek M. Antibiotic resistance of canine Staphylococcus intermedius group (SIG) - practical implications. Pol J Vet Sci. 2011;14(2):213-218.

2. Fazakerley J, Nuttall T, Sales D, et al. Staphylococcal colonization of mucosal and lesional skin sites in atopic and healthy dogs. Vet Dermatol. 2009;20(3):179-184

3. Marsella R, Olivry T, Carlotti DN; International Task Force on Canine Atopic Dermatitis. Current evidence of skin barrier dysfunction in human and canine atopic dermatitis. Vet Dermatol. 2011;22(3):239-248. 
4. Olivry T, Foster AP, Mueller RS, McEwan NA, Chesney C, Williams HC. Interventions for atopic dermatitis in dogs: a systematic review of randomized controlled trials. Vet Dermatol. 2010;21(1):4-22.

5. Olivry T, DeBoer DJ, Favrot C, et al. Treatment of canine atopic dermatitis: 2010 clinical practice guidelines from the International Task Force on Canine Atopic Dermatitis. Vet Dermatol. 2010;21(3):233-248.

6. Olivry T, Deboer DJ, Prélaud P, Bensignor E; International Task Force on Canine Atopic Dermatitis. Food for thought: pondering the relationship between canine atopic dermatitis and -cutaneous adverse food reactions. Vet Dermatol. 2007;18(6):390-391.

7. Roguedas-Contios AM, Misery L. What is intrinsic atopic dermatitis? Clin Rev Allergy Immunol. 2011;41(3):233-236.

8. Tarpataki N. Recent developments in canine atopic dermatitis: a review. Acta Vet Hung. 2006;54(4):473-484.

9. Nuttall T, Mueller R, Bensignor E, et al. Efficacy of a $0.0584 \%$ hydrocortisone aceponate spray in the management of canine atopic dermatitis: a randomised, double blind, placebo-controlled trial. Vet Dermatol. 2009;20(3):191-198.

10. Nuttall TJ, McEwan NA, Bensignor E, Cornegliani L, Löwenstein C, Rème CA. Comparable efficacy of a topical $0.0584 \%$ hydrocortisone aceponate spray and oral ciclosporin in treating canine atopic dermatitis. Vet Dermatol. 2012;23(1):4-10.

11. Deboer DJ, Schafer JH, Salsbury CS, et al. Multiple-center study of reduced-concentration triamcinolone topical solution for the treatment of dogs with known or suspected allergic pruritus. Am J Vet Res. 2002;63(3):408-413.

12. Schilling J, Mueller RS. Double-blinded, placebo-controlled study to evaluate an antipruritic shampoo for dogs with allergic pruritus. Vet Rec. June 7, 2012. [Epub ahead of print.]

13. Marsella R, Nicklin C, Lopez J. Studies on the role of routes of allergen exposure in high IgE-producing beagle dogs sensitized to house dust mites. Vet Dermatol. 2006;17(5):306-312.

14. Löflath A, von Voigts-Rhetz A, Jaeger K, et al. The efficacy of a commercial shampoo and whirlpooling in the treatment of canine pruritus - a double-blinded, randomized, placebo-controlled study. Vet Dermatol. 2007;18(6):427-431.

15. Fritz SA, Camins BC, Eisenstein KA, et al. Effectiveness of measures to eradicate Staphylococcus aureus carriage in patients with communityassociated skin and soft-tissue infections: a randomized trial. Infect Control Hosp Epidemiol. 2011;32(9):872-880.

16. Craig FE, Smith EV, Williams HC. Bleach baths to reduce severity of atopic dermatitis colonized by Staphylococcus. Arch Dermatol. 2010;146(5):541-543.

17. Huang JT, Rademaker A, Paller AS. Dilute bleach baths for Staphylococcus aureus colonization in atopic dermatitis to decrease disease severity. Arch Dermatol. 2011;147(2):246-247.

18. Zur G, White SD, Ihrke PJ, Kass PH, Toebe N. Canine atopic dermatitis: a retrospective study of 169 cases examined at the University of California, Davis, 1992-1998. Part II. Response to hyposensitization. Vet Dermatol. 2002;13(2):103-111.

19. Colombo S, Hill PB, Shaw DJ, Thoday KL. Requirement for additional treatment for dogs with atopic dermatitis undergoing allergen-specific immunotherapy. Vet Rec. 2007;160(25):861-864.

20. Marsella R, Ahrens K. Investigations on the effects of sublingual immunotherapy on clinical signs and immunological parameters using a canine model of atopic dermatitis: a double blinded, randomized, controlled study. Proceedings of the 7th World Congress, Vancouver, Canada, 2012. To be published on the Advances of Veterinary Dermatology.

21. Colombo S, Hill PB, Shaw DJ, Thoday KL. Effectiveness of low dose immunotherapy in the treatment of canine atopic dermatitis: a prospective, double-blinded, clinical study. Vet Dermatol. 2005;16(3):162-170.

22. Olivry T, Mueller RS; International Task Force on Canine Atopic Dermatitis. Evidence-based veterinary dermatology: a systematic review of the pharmacotherapy of canine atopic dermatitis. Vet Dermatol. 2003;14(3):121-146.
23. Dell DL, Griffin CE, Thompson LA, Griffies JD. Owner assessment of therapeutic interventions for canine atopic dermatitis: a long-term retrospective analysis. Vet Dermatol. 2012;23(3):228-e47.

24. Popa I, Pin D, Remoué N, et al. Analysis of epidermal lipids in normal and atopic dogs, before and after administration of an oral omega-6/ omega-3 fatty acid feed supplement. A pilot study. Vet Res Commun. 2011;35:501-509.

25. Singh SK, Dimri U, Saxena SK, Jadhav RK. Therapeutic management of canine atopic dermatitis by combination of pentoxifylline and PUFAs. J Vet Pharmacol Ther. 2010;33(5):495-498.

26. Marsella R, Nicklin CF. Double blinded placebo-controlled, cross-over study on the efficacy of pentoxifylline for canine atopy. Vet Dermatol. 2000;11(4):255-260.

27. Wirén K, Nohlgård C, Nyberg F, et al. Treatment with a barrierstrengthening moisturizing cream delays relapse of atopic dermatitis: a prospective and randomized controlled clinical trial. J Eur Acad Dermatol Venereol. 2009;23:1267-1272.

28. Marsella R, Samuelson D, Doerr K. Transmission electron microscopy studies in an experimental model of canine atopic dermatitis. Vet Dermatol. 2010;21:81-88.

29. Yoon JS, Nishifuji K, Sasaki A, et al. Alteration of stratum corneum ceramide profiles in spontaneous canine model of atopic dermatitis. Exp Dermatol. 2011;20:732-736.

30. Reiter LV, Torres SM, Wertz PW. Characterization and quantification of ceramides in the nonlesional skin of canine patients with atopic dermatitis compared with controls. Vet Dermatol. 2009;20: 260-266.

31. Shimada K, Yoon JS, Yoshihara T, et al. Increased transepidermal water loss and decreased ceramide content in lesional and non-lesional skin of dogs with atopic dermatitis. Vet Dermatol. 2009;20:541-546.

32. Marsella R. Are transepidermal water loss and clinical signs correlated in canine atopic dermatitis? A compilation of studies. Vet Dermatol. 2012;23(3):238-e49.

33. Lau-Gillard PJ, Hill PB, Chesney CJ, et al. Evaluation of a hand-held evaporimeter (VapoMeter) for the measurement of transepidermal water loss in healthy dogs. Vet Dermatol. 2010;21:136-145.

34. Popa I, Remoue N, Osta B, et al. The lipid alterations in the stratum corneum of dogs with atopic dermatitis are alleviated by topical application of a sphingolipid-containing emulsion. Clin Exp Dermatol. 2012;37(6):665-671.

35. Fujimura M, Nakatsuji Y, Fujiwara S, Rème C, Gatto H. Spot-on skin lipid complex as an adjunct therapy in dogs with atopic dermatitis: an open pilot study. Vet Med Int. 2011;2011: Article ID 281846.

36. Marsella R, Genovese D, Gilmer L, Ahrens K. Investigations on the effects of a topical ceramide and free fatty acid solution (Allerderm Spot On) on clinical signs and skin barrier function in dogs with atopic dermatitis: a double blinded, randomized, controlled study. In the proceedings of the 7th World Congress of Veterinary Dermatology, Vancouver, Canada, 2012. To be published on the Advances of Veterinary Dermatology.

37. Kircik LH, Del Rosso JQ. Nonsteroidal treatment of atopic dermatitis in pediatric patients with a ceramide-dominant topical emulsion formulated with an optimized ratio of physiological lipids. J Clin Aesthet Dermatol. 2011;4:25-31.

38. Madaan A. Epiceram for the treatment of atopic dermatitis. Drugs Today (Barc). 2008;44:751-755.

39. Sugarman JL, Parish LC. Efficacy of a lipid-based barrier repair formulation in moderate-to-severe pediatric atopic dermatitis. J Drugs Dermatol. 2009;8:1106-1111.

40. Bourdeau P, Bruet V, Gremillet C. Evaluation of phytosphingosinecontaining shampoo and microemulsion spray in the clinical control of allergic dermatoses in dogs: preliminary results of a multicentre study. Vet Dermatol. 2007;18:177-178.

41. Tokura Y. Extrinsic and intrinsic types of atopic dermatitis. J Dermatol Sci. 2010;58(1):1-7.

42. Fujimura M. The study of canine atopic dermatitis involving the isolation of dogs. Pol J Vet Sci. 2011;14(2):273-277. 
43. Rautava S, Ruuskanen O, Ouwehand A, Salminen S, Isolauri E. The hygiene hypothesis of atopic disease - an extended version. $J$ Pediatr Gastroenterol Nutr. 2004;38(4):378-388.

44. Sheikh A, Strachan DP. The hygiene theory: fact or fiction? Curr Opin Otolaryngol Head Neck Surg. 2004;12(3):232-236.

45. Rijkers GT, Bengmark S, Enck P, et al. Guidance for substantiating the evidence for beneficial effects of probiotics: current status and recommendations for future research. J Nutr. 2010;140:671S-676S.

46. Kalliomäki M, Antoine JM, Herz U, et al. Guidance for substantiating the evidence for beneficial effects of probiotics: prevention and management of allergic diseases by probiotics. J Nutr. 2010;140:713S-721S.
47. Marsella R. Evaluation of Lactobacillus rhamnosus strain GG for the prevention of atopic dermatitis in dogs. Am J Vet Res. 2009;70(6):735-740.

48. Marsella R, Ahrens K, Santoro D. Long term clinical and immunological effects of probiotics in atopic Beagles. Vet Immunol Immunopathol. 15 2012;146(2):185-189.

Veterinary Medicine: Research and Reports

\section{Publish your work in this journal}

Veterinary Medicine: Research and Reports is an international, peer-reviewed, open access journal publishing original research, case reports, editorials, reviews and commentaries on all areas of veterinary medicine. The manuscript management system is completely online and includes a very quick and fair peer-review system.
Visit http://www.dovepress.com/testimonials.php to read real quotes from published authors.

Submit your manuscript here: http://www.dovepress.com/veterinary-medicine-research-and-reports-journal 